\title{
Co-imaging of the tumor oxygenation and Research Paper using electron paramagnetic resonance imaging and 13-C hyperpolarized magnetic resonance imaging before and after irradiation
}

\author{
Masayuki Matsuo ${ }^{1,4}$, Tatsuya Kawai ${ }^{2}$, Shun Kishimoto ${ }^{1}$, Keita Saito ${ }^{1}$, Jeeva \\ Munasinghe $^{3}$, Nallathamby Devasahayam ${ }^{1}$, James B. Mitchell ${ }^{1}$ and Murali C. \\ Krishna ${ }^{1}$ \\ ${ }^{1}$ Radiation Biology Branch, Center for Cancer research, National Cancer Institute, National Institutes of Health, Bethesda, \\ MD, USA \\ ${ }^{2}$ Radiation Oncology Branch, Center for Cancer research, National Cancer Institute, National Institutes of Health, Bethesda, \\ MD, USA \\ ${ }^{3}$ MRI Research Facility, National Institute of Neurological Disorders and Stroke, National Institutes of Health, Bethesda, MD, \\ USA \\ ${ }^{4}$ Department of Radiology, Gifu University Graduate School of Medicine, Gifu City, Japan \\ Correspondence to: Murali C. Krishna, email: murali@helix.nih.gov \\ Keywords: $\mathrm{PO}_{2}$; ESR imaging; 1-13C MRl; glycolytic metabolism; radiation therapy \\ Received: November 08, $2017 \quad$ Accepted: April 02, $2018 \quad$ Published: May 18, 2018 \\ Copyright: Matsuo et al. This is an open-access article distributed under the terms of the Creative Commons Attribution License \\ 3.0 (CC BY 3.0), which permits unrestricted use, distribution, and reproduction in any medium, provided the original author and \\ source are credited.
}

\section{ABSTRACT}

To examine the relationship between local oxygen partial pressure and energy metabolism in the tumor, electron paramagnetic resonance imaging (EPRI) and magnetic resonance imaging (MRI) with hyperpolarized $\left[1-^{13} \mathrm{C}\right]$ pyruvate were performed.

SCCVII and HT29 solid tumors implanted in the mouse leg were imaged by EPRI using OX063, a paramagnetic probe and ${ }^{13} \mathrm{C}-\mathrm{MRI}$ using hyperpolarized $\left[1{ }^{13} \mathrm{C}\right]$ pyruvate. Local partial oxygen pressure and pyruvate metabolism in the two tumor implants were examined. The effect of a single dose of 5-Gy irradiation on the $\mathrm{pO}_{2}$ and metabolism was also investigated by sequential imaging of EPRI and ${ }^{13} \mathrm{C}-\mathrm{MRI}$ in HT29 tumors.

A phantom study using tubes filled with different concentration of $\left[1-{ }^{13} \mathrm{C}\right]$ pyruvate, $\left[1-{ }^{13} \mathrm{C}\right]$ lactate, and $0 \times 063$ at different levels of oxygen confirmed the validity of this sequential imaging of EPRI and hyperpolarized ${ }^{13} \mathrm{C}-\mathrm{MRI}$. In vivo studies revealed SCCVII tumor had a significantly larger hypoxic fraction ( $\mathrm{pO}_{2}<8 \mathrm{mmHg}$ ) compared to HT29 tumor. The flux of pyruvate-to-lactate conversion was also higher in SCCVII than HT29. The lactate-to-pyruvate ratio in hypoxic regions $\left(\mathrm{pO}_{2}<8 \mathrm{mmHg}\right)$ 24 hours after 5-Gy irradiation was significantly higher than those without irradiation ( 0.76 vs. 0.36$)$ in HT29 tumor. The in vitro study showed an increase in extracellular acidification rate after irradiation.

In conclusion, co-imaging of $\mathrm{pO}_{2}$ and pyruvate-to-lactate conversion kinetics successfully showed the local metabolic changes especially in hypoxic area induced by radiation therapy. 


\section{INTRODUCTION}

Tissue partial pressure of oxygen $\left(\mathrm{pO}_{2}\right)$ is linked to many pathophysiological conditions (e.g., ischemic diseases, reperfusion injury, and oxygen toxicity). Oxygen deficiency or hypoxia can increase the tumor's resistance toward cancer treatments, including radiation therapy and chemotherapy $[1,2]$. Additionally, hypoxic microenvironments in tumors are known to promote processes driving malignant progression, such as neoangiogenesis, genetic instability, and metastasis [3-5]. There is a clear oxygen dependent relationship between the radiation dose delivered locally to the tumor and the response to the radiation. This finding was essentially attributed to hypoxic cells being resistant to radiation [6]. Considering the fact that many solid tumors outgrow the blood supply and, therefore, have some regions with chronic and intermittent hypoxia [7-9], investigation of both tumor oxygenation and energy metabolism simultaneously and non-invasively is required to not only understand the radiosensitivity in individual tumor but also assess the treatment efficacy of radiation therapy.

Electron paramagnetic resonance imaging (EPRI) is a spectroscopic technique similar to nuclear magnetic resonance. EPR detects paramagnetic species that have unpaired electrons such as transition metal complexes and free radicals. With the recent availability of trityl radical probes as in vivo compatible paramagnetic probes, EPRI is now being explored for mapping tissue oxygen in live animals [8-12]. Recently dynamic nuclear polarization (DNP) techniques which can polarize the nuclear spin of ${ }^{13} \mathrm{C}$-labeled substrates far beyond thermal equilibrium conditions have been devised enabling metabolic MRI $[13,14]$ to monitor specific enzymatic reactions elevated in cancer. Many studies have shown that the polarization of $\left[1-{ }^{13} \mathrm{C}\right]$ pyruvate provides sufficient MR signal for high spatial and temporal resolution spectroscopy to monitor its metabolites such as lactate catalyzed by the enzyme lactate dehydrogenase (LDH) which is elevated in cancers [15-18]. A clinical study of DNP-MRI using hyperpolarized $\left[1-{ }^{13} \mathrm{C}\right]$-pyruvate has already been demonstrated in prostate cancer $[19,20]$ and myocardium [21].

In the present study, non-invasive sequential imaging of EPRI for tumor $\mathrm{pO}_{2}$ and DNP-MRI using hyperpolarized $\left[1-{ }^{13} \mathrm{C}\right]$ pyruvate for metabolic profile was conducted to evaluate the pixel by pixel correlation between intratumor $\mathrm{pO}_{2}$ and glycolytic profile. The relationship between energy metabolism and tumor oxygen status was investigated by sequential study of metabolic imaging by ${ }^{13} \mathrm{C}-\mathrm{MRI}$ and EPR oximetry in two different tumor cell lines, SCCVII and HT29, grown as tumors in mice. In addition, the alteration of metabolic profile and $\mathrm{pO}_{2}$ status in response to 5-Gy irradiation in HT29 tumor was examined in the same manner. These results suggested that multimodal imaging would serve as a non-invasive means of studying tumor physiology and metabolism to comprehensively monitor the tumor microenvironment and associated changes in response to treatment.

\section{RESULTS}

\section{Validation of serial imaging of $\mathrm{pO}_{2}$ and ${ }^{13} \mathrm{C}$-MRI}

To validate the ability of EPRI to provide images of absolute oxygen concentration and distinguish various levels of $\mathrm{pO}_{2}$, tubes filled with identical concentration of the paramagnetic probe, OX063 equilibrated with different levels of oxygen was studied. Figure 1A-1C show the intensity maps from the EPRI representing the concentration of OX063 and Figure 1D-1F show the corresponding maps of $\mathrm{pO}_{2}$. From the images, it can be seen that EPRI generated maps of $\mathrm{pO}_{2}$ validly represent the oxygen concentration in the solutions. T2 weighted MRI images and ${ }^{13} \mathrm{C}$ images of tubes filled with aqueous solutions of pyruvate alone (Figure 1G, 1J, 1M), lactate alone (Figure 1H, 1K, 1N) and pyruvate + lactate (Figure $1 \mathrm{I}, 1 \mathrm{~L}, 1 \mathrm{O})$ show that it is possible to distinguish pyruvate and lactate and the combination using ${ }^{13} \mathrm{C}$-MRI. The phantom study supports the capability of the sequential image of EPR and ${ }^{13} \mathrm{C}$-MRI to monitor $\mathrm{pO}_{2}$ and metabolism.

\section{In vivo study}

The feasibility of co-imaging of tissue $\mathrm{pO}_{2}$ and metabolic ${ }^{13} \mathrm{C}$ MRI in vivo was evaluated in a mouse implanted with SCCVII tumor on the leg. The absolute $\mathrm{pO}_{2}$ map from EPRI of the SCCVII tumor clearly shows a hypoxic region in the center of the tumor (Figure 2A, blue color) surrounded by a normoxic rim. After EPRI acquisition, the same mouse was transferred to a 7 T MRI for anatomic and metabolic imaging. Figure $2 \mathrm{~B}$ shows the anatomic image of the tumor overlaid with ${ }^{13} \mathrm{C}$ spectra obtained $12 \mathrm{sec}$ after hyperpolarized $\left[1-{ }^{13} \mathrm{C}\right]$ pyruvate injection. $\left[1-{ }^{13} \mathrm{C}\right]$ Pyruvate (right peak in the spectra) and $\left[1-{ }^{13} \mathrm{C}\right]$ lactate (left peak) were detectable in each of tumor regions. A lactate-to-pyruvate ratio map (Figure 2C) calculated from the ${ }^{13} \mathrm{C}$ spectroscopic images showed heterogeneous distribution of these metabolites within the tumor regions and relatively high lactate/pyruvate ratio was observed in the areas spatially overlapped with hypoxic regions. (Figure 2A and 2C).

\section{Comparison of tumor $\mathrm{pO}_{2}$ and pyruvate metabolism in two cancer cell lines}

Typically, the tumor size largely influences the tumor physiology and the outcome of cancer therapy. Previous studies reported that tumor oxygenation and vascularity decreased with an increase of tumor size $[22,23]$. Therefore, the tumor oxygenation and pyruvate metabolism in two cancer cell lines were compared when 
they were almost the same in size. The tumor size of $\operatorname{SCCVII}(n=4)$ was $643 \pm 37($ mean $\pm \operatorname{SEM}) \mathrm{mm}^{3}$ and that of HT29 tumor $(n=6)$ was $627 \pm 17 \mathrm{~mm}^{3}$. Although the median tumor $\mathrm{pO}_{2}$ of SCCVII was $13.5 \mathrm{mmHg}$, lower than that of the HT29 tumor $(15.8 \mathrm{mmHg})$, the difference was not significant. Detailed analyses of $\mathrm{pO}_{2}$ fractions revealed that the hypoxic regions with $\mathrm{pO}_{2}<8 \mathrm{mmHg}$ in SCCVII tumor was $18.5 \%$ which was significantly larger than that of the HT29 tumor $(3.3 \%, P<0.01)$. On the other hand, there was no significant difference between the fraction of normoxic region with $\mathrm{pO}_{2}>16 \mathrm{mmHg}$ in SCCVII and HT29 tumor (32.6\% and 49.9\%, respectively) (Figure 3A). These results are consistent with previous findings from histological data for hypoxia using pimonidazole [9].
Figure $3 \mathrm{~B}$ shows a typical time course of ${ }^{13} \mathrm{C}-\mathrm{MR}$ spectroscopy obtained after the EPRI of $\left[1-{ }^{13} \mathrm{C}\right]$ pyruvate and its metabolic product $\left[1-{ }^{13} \mathrm{C}\right]$ lactate in SCCVII and HT29 tumor. A strong lactate signal buildup was observed immediately in both tumors after the pyruvate signal in tumor region, suggesting that intravenously injected pyruvate was taken up into the cells and enzymatically converted into lactate in the tumors. Hence, the time to peak of lactate signal intensity was observed later than that of pyruvate signal both in SCCVII (Supplementary Figure 1) and HT29 tumor (Supplementary Figure 2). Figure 3C shows $\left[1-{ }^{13} \mathrm{C}\right]$ pyruvate and $\left[1-{ }^{13} \mathrm{C}\right]$ lactate signal intensity in SCCVII and HT29 tumor calculated from area under curve of the time-intensity profile of the dynamic MR

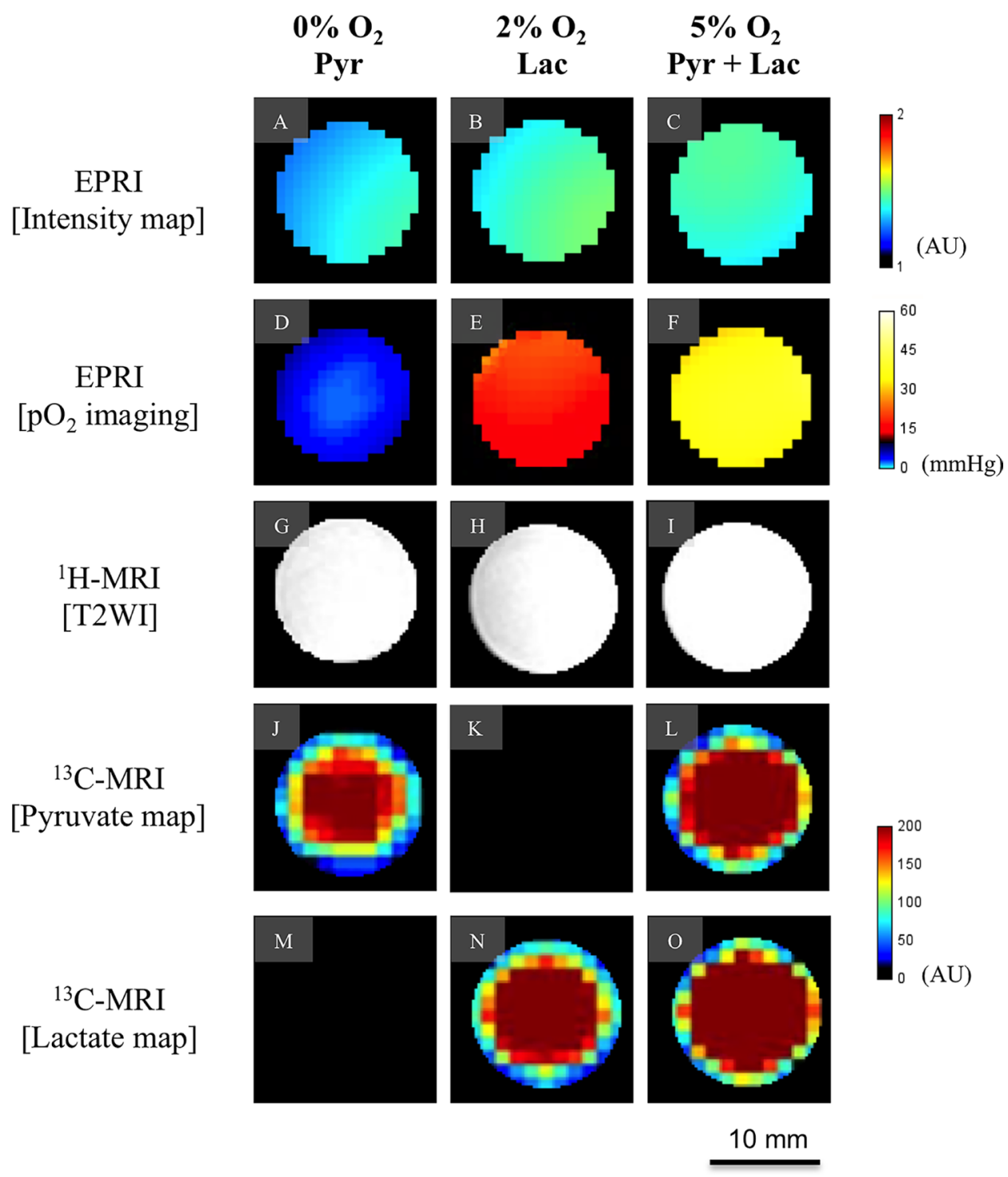

Figure 1: Validation of EPR oxygen imaging and [1-13C] Metabolic MRI in phantom. A phantom object with three-tube of aqueous solutions containing $3 \mathrm{mM}$ of the oxygen sensitive paramagnetic tracer OX063 equilibrated with $0 \%$ oxygen and $1 \mathrm{M}$ [1-13C] pyruvate (left columun), $2 \%$ oxygen and $1 \mathrm{M}$ [1-13C] lactate (middle column), and 5\% oxygen and $1 \mathrm{M}$ [1-13C] pyruvate and $1 \mathrm{M}$ [1-13C] lactate (right column). Each row shows signal intensity map on EPRI (A-C), pO 2 map on EPRI (D-F), T2-weighted image on ${ }^{1} \mathrm{H}-\mathrm{MRI}$ $(\mathbf{G}-\mathbf{I})$, pyruvate map on ${ }^{13} \mathrm{C}$-MRI $(\mathbf{J}-\mathbf{L})$, and lactate map on ${ }^{13} \mathrm{C}$-MRI $(\mathbf{M}-\mathbf{O})$, respectively. Color-coded indicators are shown on the right for EPRI and ${ }^{13} \mathrm{C}-\mathrm{MRI}$. 
spectroscopy obtained between 0 and 80 seconds. Total pyruvate in HT29 tumor was higher than that of SCCVII tumor. The total signal of pyruvate in HT29 tumor was significantly higher than lactate, whereas there was no significant difference in SCCVII tumor. On the other hand, lactate-to-pyruvate ratio calculated from the signal intensity between the 20 and 40 second in SCCVII and HT29 tumor showed that it was significantly higher in SCCVII than HT29 (Figure 3D). As a previous report form Yasui et al. [9] showed a remarkable fluctuation of $\mathrm{pO}_{2}$ within a few minutes in the SCCVII compared to HT29, where it was shown more stable. Since the main aim of our study was co-imaging of tumor hypoxia and metabolism, we selected only HT29 for co-imaging study using EPRI and 13C-MRI under a hypothesis that the oxygen status in the tumor was almost the same between the time points when the two imaging were performed.

\section{Hypoxic tumor sub-region increases post radiation}

The effect of 5 Gy irradiation on tumor oxygenation was investigated by EPR oxygen imaging. Averaged tumor size in the 5 Gy-treated group $(n=6)$ was $639 \pm 26 \mathrm{~mm}^{3}$ (mean \pm SEM) and there was no significant difference statistically from that of non-treated control group (627 $\pm 17 \mathrm{~mm}^{3}, n=6$ ). Although there was no significant difference in averaged $\mathrm{pO}_{2}$ over the whole tumor regions between non-treated $(15.8 \mathrm{mmHg})$ and the 5 Gy-treated groups $(15.2 \mathrm{mmHg})$, the fractional hypoxic volume $\left(\mathrm{pO}_{2}<8 \mathrm{mmHg}\right)$ in the 5 Gy-treated group (15.4\%) was significantly higher than non-treated control group (3.2\%). The fractional normoxic volume (with $\mathrm{pO}_{2}>16 \mathrm{mmHg}$ ) of non-treated and the 5-Gy treated groups were 50.5\% and $55.3 \%$, respectively (Figure $4 \mathrm{~A}$ ).

\section{Gy irradiation suppresses tumor perfusion and enhances flux of pyruvate-to-lactate conversion in hypoxic tumor sub-regions}

Outcome of radiation therapy is known to depend on tumor oxygen status [2]. However post-radiation changes in the tumor metabolism are not known. We investigated the effect of a single 5-Gy irradiation on the relationship between tumor $\mathrm{pO}_{2}$ and energy metabolism by sequential imaging with EPRI and hyperpolarized ${ }^{13} \mathrm{C}$-MRI. Supplementary Figure 2 shows time-to-intensity curves of ${ }^{13} \mathrm{C}$-lactate and pyruvate in non-treated control and
A

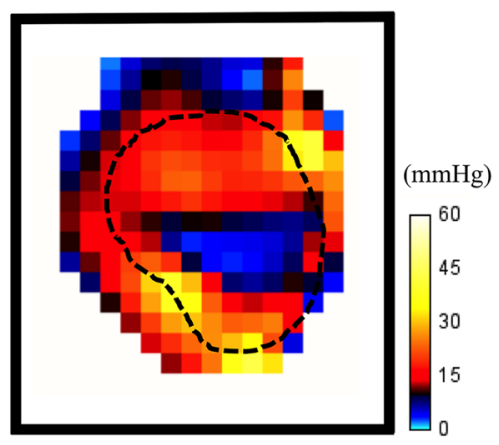

$\mathrm{pO}_{2}$ mapping

B

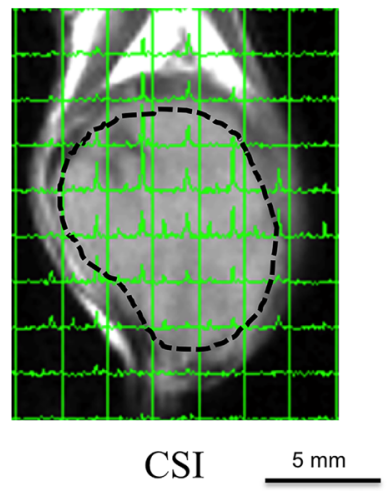

$\mathrm{C}$

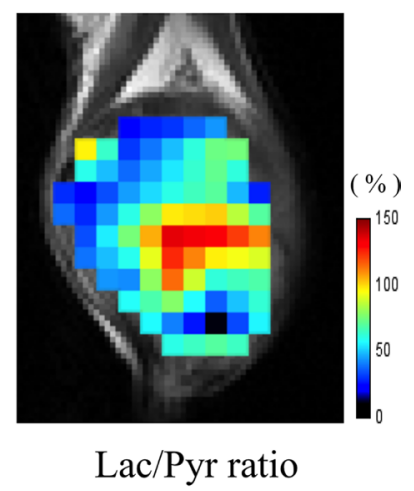

Figure 2: Feasibility of co-imaging of EPR oxygen imaging and ${ }^{13} \mathrm{C}-\mathrm{MRI}$ in vivo. An example case of co-imaging of tissue EPRI $\mathrm{pO}_{2}$ mapping and ${ }^{13} \mathrm{C}$-MRI imaging in a mouse bearing subcutaneously SCCVII tumor on the leg. (A) The absolute $\mathrm{pO}_{2}$ map shows a hypoxic core (blue) in the central part of the tumor. (B) The ${ }^{13} \mathrm{C}$-chemical shift imaging (CSI) obtained 12 sec after hyperpolarized $\left[1-{ }^{13} \mathrm{C}\right]$ pyruvate injection overlaid on the T2-weighted image. Each signal peak corresponds to $\left[1-{ }^{13} \mathrm{C}\right]$ pyruvate (right) and $\left[1-{ }^{13} \mathrm{C}\right]$ lactate $($ left), respectively. (C) Lac/Pyr ratio map. Note that the higher lactate-to-pyruvate ratio area dominantly exists in the hypoxic region on EPRI. 
24 hours after 5-Gy X-irradiated groups of mice bearing HT29 tumor, grouped into five sub-regions with different $\mathrm{pO}_{2}(<8,8-12,12-16,16-20$, and $>20 \mathrm{mmHg})$. The time to peak intensity of pyruvate signal was almost the same in all the sub-regions in both non-treated and the 5-Gy irradiated groups, whereas that of lactate signal was 20 and 30 seconds in non-treated and the 5-Gy treated groups, respectively. There was no obvious $\mathrm{pO}_{2}$ dependency observed in these time courses of pyruvate and lactate signal intensity in non-treated control tumors, however, the difference of a time course of these signal intensity was clearly observed in the 5-Gy treated group dependent on their $\mathrm{pO}_{2}$ levels.

Figure $4 \mathrm{~B}$ shows total carbon $\left({ }^{13} \mathrm{C}\right.$-pyruvate + ${ }^{13} \mathrm{C}$-lactate) signal intensity calculated from the time-tointensity curve from 0 to 80 seconds, a total of 20 images in non-irradiated 24 hours after 5-Gy irradiated HT29 tumor. The total carbon signal at 24 hours after 5-Gy

A

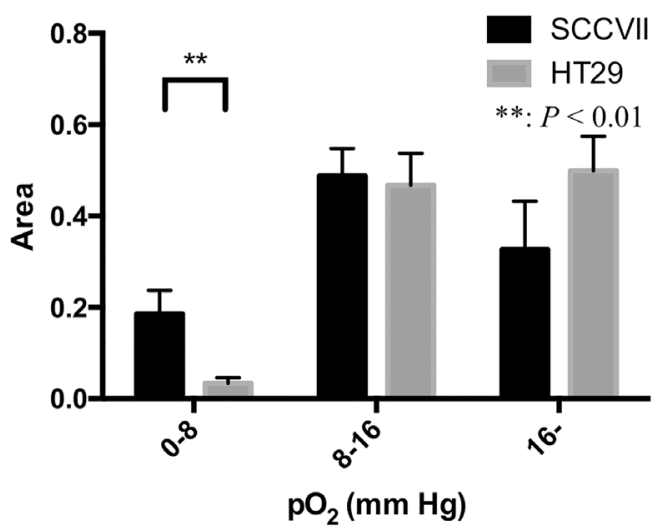

C

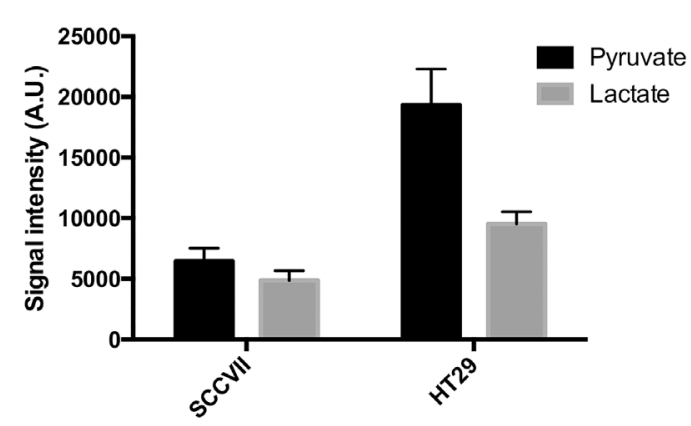

irradiation of HT29 tumor was significantly lower than that of non-treated and 24 hours after 5-Gy irradiation (0-20 mmHg). Difference in the total carbon between nontreated control and 24 hours after 5-Gy irradiated tumors was largest in hypoxic tumor regions with $\mathrm{pO}_{2}<8 \mathrm{mmHg}$, and smallest in normoxic sub-regions with $\mathrm{pO}_{2}>$ $20 \mathrm{mmHg}$. The decrease in total ${ }^{13} \mathrm{C}$ signal in the treated group implies decrease in tumor blood perfusion, which was further confirmed by decrease in Gd-DTPA uptake during the first 1 min after injection in DCE-MRI study (Supplementary Figure 3).

Figure 4C shows lactate-to-pyruvate ratio calculated from the time-to-intensity curve from 20 to 40 seconds in non-irradiated and 24 hours after a 5-Gy irradiated HT29 tumor. Lactate-to-pyruvate ratio in $0-8 \mathrm{mmHg}$ at 24 hours after 5-Gy irradiation of HT29 tumor $(76 \%)$ is higher $(P<0.01)$ than of non irradiation of HT29 tumor $(36 \%)$, suggesting that the enzymatic conversion of pyruvate to

B

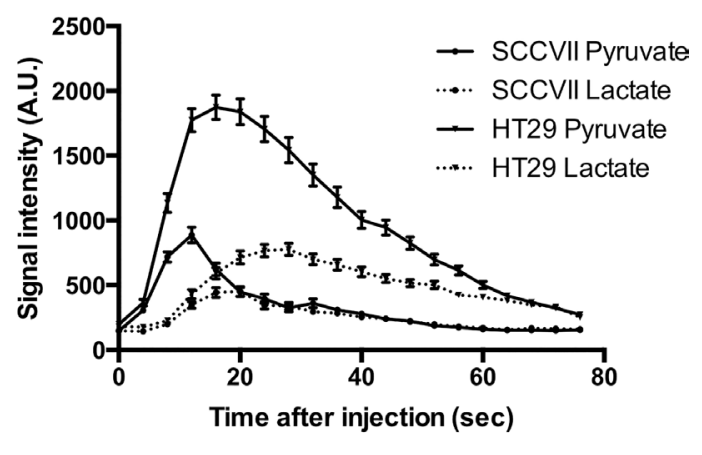

$\mathrm{D}$

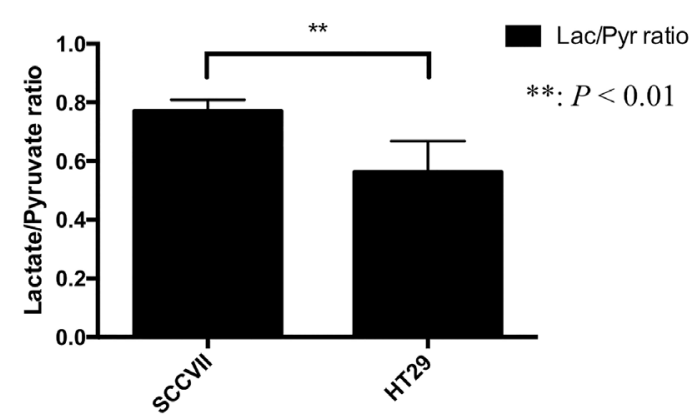

Figure 3: Tumor volume, $\mathrm{pO}_{2}, \mathrm{pO}_{2}$ fraction, and Glycolytic metabolism in SCCVII and HT29 tumor. (A) The difference in three $\mathrm{pO}_{2}$ fractional areas $(0-8,8-16$ and $>16 \mathrm{mmHg})$ between SCCVII $(n=4)$ and HT29 $(n=6)$ tumors. (B) The time courses of ${ }^{13} \mathrm{C}$ MR signal intensity of $\left[1-{ }^{13} \mathrm{C}\right]$ pyruvate and its metabolic product $\left[1-{ }^{13} \mathrm{C}\right]$ lactate in SCCVII and HT29 tumor. (C) The sum of pyruvate and lactate signal intensity calculated from 20 images acquired during the first 80 seconds after an injection of $\left[1-{ }^{13} \mathrm{C}\right]$ pyruvate. (D). Lactateto-pyruvate ratio in SCCVII calculated from a time course from 20 to 40 seconds. Statistically significant differences are indicated with asterisks. 
lactate was much higher 24 hours after 5-Gy irradiation in the area with $0-8 \mathrm{mmHg}$, especially under $16 \mathrm{mmHg}$.

\section{In vitro study}

\section{Extracellular acidification rate (ECAR) as a maker of lactate production}

To confirm the increase in lactate-to-pyruvate ratio after irradiation on ${ }^{13} \mathrm{C}$-MRI, we conducted an in vitro study independently, where lactate production form the tumor cells were assessed. In this experiment, subcutaneous HT29 tumors were excised $24 \mathrm{~h}$ after 0 , 3,5 , or $10-$ Gy irradiation followed by homogenization and trypsin-incubation to prepare single cells. Using $1 \times 10^{4}$ cells in each well of a 96-well plate, extracellular acidification rate (ECAR) was measured by SeaHorse extracellular flux analyzer as a maker of lactate production. Figure 5A shows a time course of ECAR in the culture media with the cells treated by $0,3,5$, or $10 \mathrm{~Gy}$ and Figure 5B shows AUCs of ECAR. A significant

A
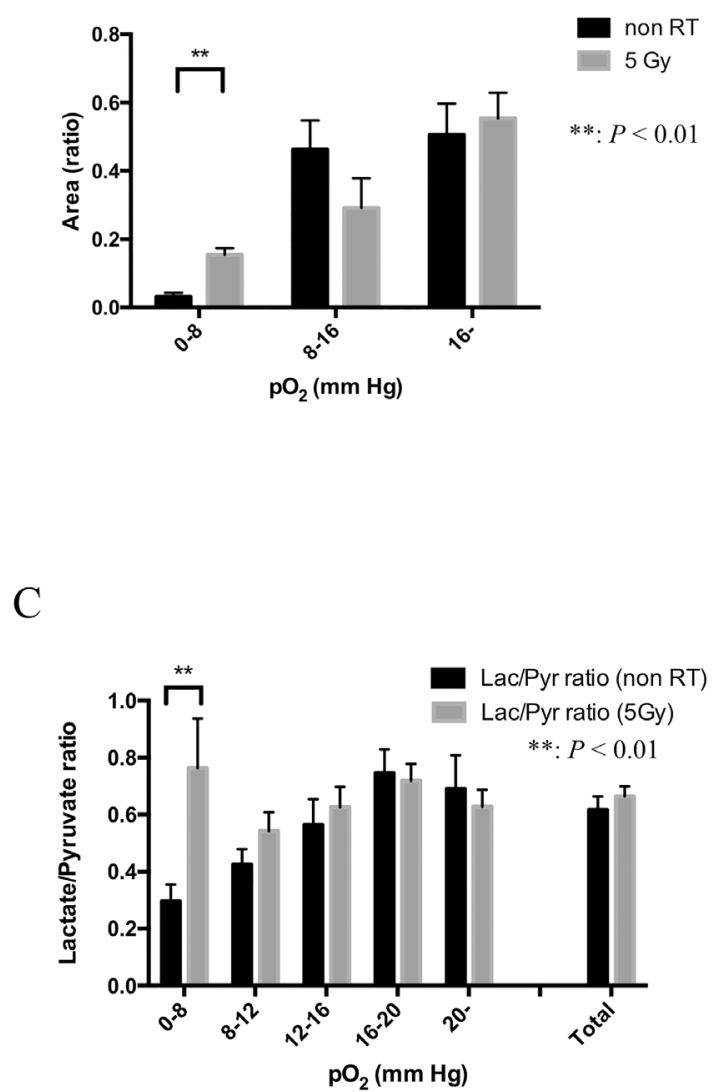

difference between the increase in ECAR in the nonirradiated control group and that in the 5-Gy irradiation group (192.0 vs $630.3 \mathrm{mpH} / 10^{4}$ cells) was observed after an injection of ATP synthase inhibitor, oligomycin. The increase in ECAR of 10-Gy treated cells was smaller than 5-Gy treated cells presumably as a result of substantial damage of the cells.

\section{DISCUSSION}

Technological developments in medical physics for the precise delivery of radiation dose has been paralleled in recent years by a greatly-improved molecular imaging techniques which can profile the tumor microenvironment in terms of physiology band metabolism. To take full advantage of the curative potential of modern and modern radiotherapy techniques such as stereotactic radiotherapy, radiosurgery, and intensity-modulated radiotherapy (IMRT), molecular imaging to profile the microenvironment is needed for identification of target volumes [24-28]. Recently, the development of these

B

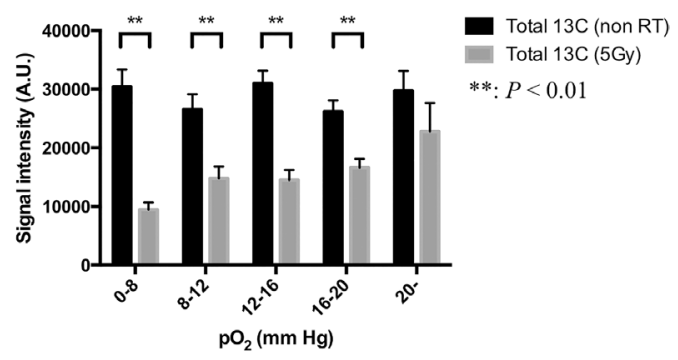

Figure 4: Glycolytic metabolism in each $\mathrm{pO}_{2}$ sub-region: comparison between non-irradiation control and 24 hours after 5-Gy irradiation in HT29 tumor. (A) The difference in three $\mathrm{pO}_{2}$ fractional areas $(0-8,8-16$ and $>16 \mathrm{mmHg})$ between 5-Gy irradiated group $(n=6)$ and control group $(n=6)$. (B) The sum of the total carbon $\left(\left[1-{ }^{13} \mathrm{C}\right]\right.$ pyruvate and $\left[1-{ }^{13} \mathrm{C}\right]$ lactate) signal intensity calculated from 20 images acquired during the first 80 seconds after an injection of $\left[1-{ }^{13} \mathrm{C}\right]$ pyruvate in five different $\mathrm{pO}_{2}$ fractional areas $(0-$ $8,8-12,12-16,16-20$ and $>20 \mathrm{mmHg}$ ) in the two groups. (C). Lactate-to-pyruvate ratio calculated from a time course from 20 to 40 seconds in non-irradiation control group and 24 hours after 5-Gy irradiated group. Statistically significant differences are indicated with asterisks. 
technologies has been drawing clinicians' attention to molecular imaging for prospect of highly targeted radiation therapy and providing new research opportunities in preclinical and clinical study in small-animal models. Tumor blood flow is one of the important biological parameters in the cancer therapy because it would affect not only drug distribution but also tumor hypoxia as a result of impaired blood flow that may cause radiation resistance. Therefore, molecular imaging techniques which enables us to assess the tumor metabolism and oxygenation state before and during its treatment is needed [23]. In this study, we utilized a system where the oxygen mapping and the metabolic imaging to be co-registered using EPRI and hyperpolarized ${ }^{13} \mathrm{C}$-MRI and successfully showed correlation between the oxygen partial pressure and lactate-pyruvate distribution on the anatomical images. First, we validated the feasibility of this method using in vitro and in vivo (Figures 1 and 2). Figure $2 \mathrm{~A}$ and Figure $2 \mathrm{C}$ showed the heterogeneous oxygen distribution and lactate-to-pyruvate ratio, respectively, in the same representative SCCVII tumor. They also described the regions with high lactate-to-pyruvate ratio spatially concordant with the regions with hypoxia. The differences in tumor hypoxic state and pyruvate metabolism between SCCVII and HT29 tumor were clearly observed. Since majority of signal peaks observed immediately after the pyruvate signal peak on hyperpolarized ${ }^{13} \mathrm{C}$-MRI was from lactate, we also estimated the blood flow volume using a summation of peak signal intensity of pyruvate and lactate as the approximate total signal from carbon-13. There was a significant difference in the signal intensity from total carbon between SCCVII and HT29 tumor, suggesting lower perfusion in SCCVII tumor than HT29 (Figure 3C). The longer time-to-peak both for pyruvate and lactate after injection in SCCVII was consistent with this observation (Figure 3B). Therefore, the higher lactate-to-pyruvate ratio observed in SCCVII than HT29 as shown in Figure 3D was consistent with higher hypoxic fraction ( $\mathrm{pO} 2 \leq 8 \mathrm{mmHg}$ ) in SCCVII presumably due

A

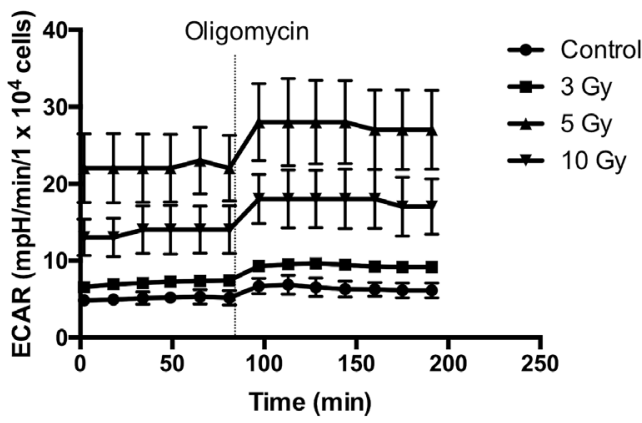

to higher LDH activity under anaerobic glycolytic state (Figure 3A). Yasui et al. [9] showed that the number of pericytes that cover blood vessels within SCCVII tumors was relatively small compared with the HT29 tumors and this difference could contribute to the difference not only in blood perfusion but also in $\mathrm{O} 2$ diffusion. As Park et al. [29] had shown decrease in tumor blood perfusion after radiation therapy, we developed a co-imaging technique to investigate changes in oxygen distribution and glycolytic metabolism modifications as a result of radiation exposure using HT29 tumor, which Yasui et al. [9] had reported relatively stable in fluctuation in oxygen status compared to SCCVII. A dynamic contrast enhanced MRI study using gadolinium contrast media is one of the well-known methods for analyses of blood perfusion in tumors and has been used to measure several relevant parameters, the microvasculature volume, vascular permeability and blood flow in various tissues [30, 31]. We confirmed that gadolinium concentration in the early phase, which reflected blood perfusion, at 24 hours after 5-Gy irradiation in HT29 tumor was lower than non-RT group (Supplementary Figure 3). This result suggested a decrease in blood flow volume resulting radiation treatment. An increase in hypoxic area $\left(\mathrm{pO}_{2}<8 \mathrm{mmHg}\right)$ by over 5 -fold was observed 24 hours after 5-Gy irradiation (Figure 4A) whereas a significant decrease in total carbon-13 signal was observed not only in hypoxic area but also in the more oxygenated areas (8-20 $\mathrm{mmHg}$ ) (Figure 4B), indicating the radiation-induced hypoxia was attributed to a global decrease in the blood flow. It is notable that there was no significant difference in total carbon-13 signal before irradiation among all of the areas including hypoxic area. This is probably because the hypoxic region in HT29 tumor before irradiation was so small that carbon-13 signals from this area could be buried with those from the surrounding more oxygenated tissue because of the thickness of FOV in ${ }^{13} \mathrm{C}$-MRI larger than EPRI ( $8 \mathrm{~mm}$ vs. $2 \mathrm{~mm}$, respectively). Likewise, a significant increase in the lactate-to-pyruvate ratio by more than 2 -fold observed in

B

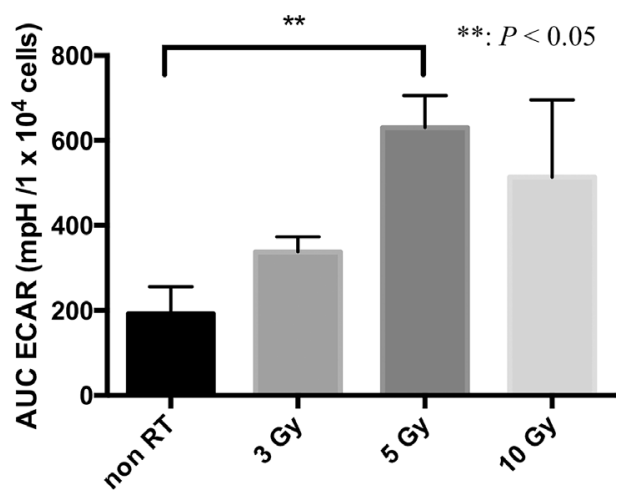

Figure 5: Extracellular acidification rate (ECAR) as a marker of lactate production. (A) The time course of ECAR in control, 3-, 5-, and 10-Gy irradiated cells in tissue culture. (B) The area under curve (AUC) of ECAR corrected by the cell numbers after an administration of oligomycin. Statistically significant differences are indicated with asterisks. 
the hypoxic area 24 hours after irradiation could be at least partially explained by hypoxic regions newly developed by radiation-induced hypoperfusion area where anaerobic glycolysis dominates grew large enough to bring the ${ }^{13} \mathrm{C}$ signal detectable.

HIF-1 $\alpha$, which is known to be stabilized when $\mathrm{O}_{2}$ availability is decreased to regulate transcription of hundreds of genes that encode proteins including pyruvate dehydrogenase kinase-1 (PDK1) and lactate dehydrogenase (LDH) [32-35]. Phosphorylation of pyruvate dehydrogenase by PDK1 reduces flux through the TCA cycle, as a result, down-regulates aerobic respiration. Pyruvate accumulated in hypoxic cells is then converted into lactate via $\mathrm{LDH}$ [36]. In our ex vivo experiment, enhanced glycolytic reserve was indicated by a significant increase in ECAR after irradiation (Figure 5). These measurements were performed at relatively higher and uniform oxygen $(21 \%)$ conditions in contrast to solid tumors. Kim et al. [33] reported hypoxia-independent stabilization of HIF-1a by a fractionated 6-Gy irradiation in glioma cell lines, suggesting there could be radiationinduced metabolic change independent on alteration of blood perfusion. Combining these findings, although still speculative, the increase in pyruvate-to-lactate ratio in the hypoxic region observed in ${ }^{13} \mathrm{C}$-MRI after irradiation might be also attributed to radiation-induced HIF-1a stabilization in addition to hypoperfusion.

In this study, we successfully showed the feasibility of sequential imaging of the $\mathrm{pO}_{2}$ map using EPRI in combination with a hyperpolarized ${ }^{13} \mathrm{C}$-MRI that provided valuable metabolic information including dynamic flux of pyruvate-to-lactate conversion. A limitation in this study is insufficient spatial resolution of ${ }^{13} \mathrm{C}$-MRI that could pass over small hypoxic lesions detected on EPRI. Since it needs 8 - $10 \mathrm{~mm}$ slab thickness to acquire sufficient MR signals from hyperpolarized carbon-13 while the in-plane resolution is $2 \mathrm{~mm}$. Nevertheless, developing state-of-the-art imaging methods, such as MRI and PET in combination with technical improvements in hypoxic and metabolic imaging technique based on our study would contribute to further improvement in treatment outcome by developing strategies of a highly personalized, tailored treatment utilizing targeting therapy against hypoxia and subsequent metabolism changes in the tumor using [37]. Radiation therapy alters the $\mathrm{pO}_{2}$ distribution in the tumor. The co-imaging of $\mathrm{pO}_{2}$ and metabolic imaging of pyruvate-to-lactate conversion kinetics can shed light on the local metabolic changes especially in hypoxic area induced by radiation therapy.

\section{MATERIALS AND METHODS}

\section{Ethics statement}

We carried out all our procedures in compliance with the Guide for the Care and Use of Laboratory Animal Resources (National Research Council, 1996), and experimental protocols were approved by the National Cancer Institute Animal Care and Use Committee (NCICCR-ACUC (Bethesda, USA), Protocol\# RBB- 159).

\section{Phantom model}

Since one of the purposes of this experiment was to assess the feasibility of serial imaging of $\mathrm{pO}_{2}$ and [1${ }^{13} \mathrm{C}$ ] using EPRI and MRI we conducted a phantom study first. Three identical cylinder-shaped glass container filled with ultrapure water containing $3 \mathrm{mM}$ of oxygen-sensitive tracer, triarylmethyl (TAM; methyl-tris[8-carboxy-2,2,6,6tetrakis[(2-hydroxyethyl]-benzo[1,2-d:4,5-d']bis[1,3] dithiol-4-yl] trisodium salt; OX063, GE Healthcare) were used. Each solution was equilibrated with (1) $0 \%$ oxygen and $1 \mathrm{M}\left[1-{ }^{13} \mathrm{C}\right]$ pyruvate, (2) $2 \%$ oxygen and $1 \mathrm{M}\left[1-{ }^{13} \mathrm{C}\right]$ lactate, and (3) $5 \%$ oxygen, $1 \mathrm{M}\left[1-{ }^{13} \mathrm{C}\right]$ pyruvate and $1 \mathrm{M}$ $\left[1-{ }^{13} \mathrm{C}\right]$ lactate, respectively, for both $\mathrm{pO}_{2}$ imaging by EPRI and ${ }^{13} \mathrm{C}$-MR spectroscopy along with anatomical imaging by $7 \mathrm{~T}$ MRI.

\section{Cell culture and tumor implantation}

Murine squamous cell carcinoma SCCVII and human colon cancer HT29 cell lines were tested in April 2013 by IDEXX RADIL (Columbia, MO) using a panel of microsatellite markers and authenticated. Female $\mathrm{C} 3 \mathrm{H} /$ Hen mice and athymic nude mice were supplied by the Frederick National Laboratory for Cancer Research Center (Frederick, MD). SCCVII and HT29 solid tumors were formed by a subcutaneous injection of $5 \times 10^{5}$ cells and $1 \times 10^{6}$ cells, respectively, into the right hind legs of mice as described previously [8]. The experiments were initiated when tumors grew to approximately $600-700 \mathrm{~mm}^{3}$. The tumor volume was calculated by a following approximation formula; Tumor volume $=$ length $\times$ width $\times$ height $\times 3.14 \times$ $1 / 6$. The body weight measured before the experiments was ranged from 21 to $27 \mathrm{~g}$. In the imaging of EPRI and MRI, mice were anesthetized by isoflurane inhalation (4\% for induction and $1.5 \%$ for maintaining anesthesia) in medical air $(750 \mathrm{~mL} / \mathrm{min})$ and positioned prone with their tumorbearing legs placed inside the resonator. During EPRI and MRI, the breathing rate of the mouse was monitored with a pressure transducer (SA, Instruments Inc.) and maintained at $60 \pm 10$ breaths per minute. Core body temperature was monitored by a FISO FTI-10 temperature sensor (FISO Technologies Inc., Quebec, Canada) and maintained at $37^{\circ} \mathrm{C}$ with a flow of warm air (EPRI) or water (MRI). For administration of OX063 and $\left[1-{ }^{13} \mathrm{C}\right]$ pyruvate solution, a 30 -gauge needle was cannulated into the tail vein and extended using a polyethylene tubing.

\section{EPR imaging for $\mathrm{pO}_{2}$}

Technical details of the EPR scanner and oxygen image reconstruction were described in earlier reports $[8,11,38-40]$. Parallel coil resonators tuned to 
$300 \mathrm{MHz}$ were used for EPRI and MRI because the use of radiofrequency $(\mathrm{RF})$ at $300 \mathrm{MHz}$ for EPR allows the EPR signal to be acquired deeper in the tissue of a living object. After the animal was placed in the resonator, the resonator (17 $\mathrm{mm}$ in diameter. and $17 \mathrm{~mm}$ long) was used as an identical coil for EPRI and MRI operating at $300 \mathrm{MHz}$. For EPRI experiment, OX063 was injected intravenously through a cannula placed in the tail vein. To keep the blood concentration, OX063 was given as a $1.125 \mathrm{mmol} / \mathrm{kg}$ bolus injection followed by $0.04 \mathrm{mmol} / \mathrm{kg} /$ min continuous injection [41]. EPR signals were collected following the radiofrequency excitation pulses; $60 \mathrm{~ns}$, $80 \mathrm{~W}, 70^{\circ}$ flip angle using an analog digital converter (200 M samples/s). EPR measurements were started 3 min after OX063 injection, and it took $12 \mathrm{~min}$ to obtain a data set for a 3D image. Although the in-plane spatial resolution at the acquisition was $1.8 \mathrm{~mm}$, it was digitally enhanced subsequently to $0.125 \mathrm{~mm}$ for co-registration with MRI images. Since Saito et al. [42] showed that a transient decrease in tumor oxygenation after intravenous administration of pyruvate, we underwent EPRI before ${ }^{13} \mathrm{C}$ imaging.

\section{MRI and co-registration of $\mathrm{pO}_{2}$ images with anatomic images}

After each EPRI scan, the mouse was transferred into a 7T MRI scanner without being removed from the holder to obtain the T2-weighted anatomical image. Anatomical images of the tumor-bearing leg were obtained using 7T MRI scanner controlled with ParaVision 5.0 (Bruker BioSpin MRI GmbH, Billerica, MA). After a quick assessment of the sample position by a fast lowangle shot (FLASH) pilot sequence, $\mathrm{T}_{2}$-weighted axial and coronal images were obtained using a fast spin echo sequence (RARE) with an echo time of $13 \mathrm{~ms}$, repetition time of 2,500 ms, 16 slices, RARE factor 8, and a resolution of $0.125 \times 0.125 \mathrm{~mm}^{2}$. For convenience of coregistration with EPRI, all MRI images had the same filed of view (FOV) of $32 \mathrm{~mm}$ and slice thickness of $2 \mathrm{~mm}$. Coregistration of EPRI and MRI images was accomplished using a code written in MATLAB (Mathworks) script as previously described $[8,18,43]$.

\section{DNP-MRI of $\left[1-{ }^{13} \mathrm{C}\right]$-labeled pyruvate metabolism}

After acquiring anatomical $\mathrm{T}_{2}$-weigthed $\mathrm{MRI}$ data set, hyperpolarized $\left[1-{ }^{13} \mathrm{C}\right]$ MRI studies were performed. To obtain hyperpolarized $\left[1-{ }^{13} \mathrm{C}\right]$ pyruvate, $30 \mu \mathrm{L}$ of pyruvic acid $\left(1-{ }^{13} \mathrm{C}, 99 \%\right.$, Cambridge Isotope Laboratories, Tewksbury, MA) containing $15 \mathrm{mM}$ of OX063 and $2.5 \mathrm{mM}$ of the gadolinium chelate ProHance (Bracco Diagnostics, Milano, Italy) were polarized at $3.35 \mathrm{~T}$ and $1.4 \mathrm{~K}$ in the Hypersense DNP polarizer (Oxford Instruments, Abingdon, UK), according to the manufacturer's instructions. After $1-1.5 \mathrm{~h}$, the hyperpolarized sample was rapidly dissolved in $4.5 \mathrm{~mL}$ of a superheated alkaline buffer comprising $40 \mathrm{mM}$ of 4-(2-hydroxyethyl)-1-piperazineethanesulfonic acid, 30 $\mathrm{mM}$ of $\mathrm{NaCl}$, and $100 \mathrm{mg} / \mathrm{L}$ of ethylendiaminetetraacetic acid to obtain $96 \mathrm{mM}\left[1-{ }^{13} \mathrm{C}\right]$ pyruvate solution. Appropriate quantity of $\mathrm{NaOH}$ was added to the dissolution buffer to adjust the pyruvate solution to be $\mathrm{pH}$ 7.4. Immediately after the dissolution, a bolus injection of $300 \mu \mathrm{L}$ (approximately $1.15 \mathrm{mmol} / \mathrm{kg}$ ) $\left[1-{ }^{13} \mathrm{C}\right]$ pyruvate solution was administrated intravenously through the tail vein cannula right after the dissolution [18]. For the dynamic study, ${ }^{13} \mathrm{C}$ spectra were acquired every 4 seconds using echo-planar spectroscopic imaging technique after the start of pyruvate injection from a 8 - to $12-\mathrm{mm}$ slice on the SCCVII and HT-29 for 240 seconds. The slice thickness was defined as it included most part of the tumor. ${ }^{13} \mathrm{C}$ two-dimensional spectroscopic images were also acquired 30 seconds after the start of the pyruvate injection with a $32 \times 32 \mathrm{~mm}^{2} \mathrm{FOV}$ in a $8 \mathrm{~mm}$ slice of horizontal plane through the longitudinal axis of the leg a matrix size of $16 \times 16$, spectral width of $9765.62 \mathrm{~Hz}$, repetition time of $125 \mathrm{~ms}$. The total time required to acquire each image was 19.2 seconds.

\section{Gadolinium enhanced MRI}

For T1 mapping, coronal RARE images of four slices passing through the tumor region were obtained with TR values of 300, 2000, and $6000 \mathrm{~ms}$. FLASH sequence of the same four slices was applied for dynamic contrast enhanced (DCE)-MRI using an administration of contrast agent, gadolinium (Gd)-DTPA (Magnevist, Beyer Pharmaceuticals, Berlin, Germany). The scan parameters were as follows: TE $4.00 \mathrm{~ms}$, TR $156 \mathrm{~ms}$, flip angle $45^{\circ}$, three slices, $0.50 \times 0.50 \mathrm{~mm}^{2}$ in-plane resolution, 20 -second acquisition time per each dynamic scan, and 98 times repetition. Gd-DTPA (50 $\mathrm{mM}, 5 \mu \mathrm{L} / \mathrm{g}$ body weight) was intravenously injected into the tail vein 2 min after the beginning of the fast gradient echo scan. The gadolinium concentration in the tumor tissue was calculated as described in a previous report [44].

\section{Extracellular acidification rate (ECAR)}

HT29 tumor mice were divided into four groups (control, 3, 5, and 10 Gy-irradiation, $n=3$ each group). The mice were euthanized by breathing carbon dioxide gas 24 hours after an irradiation. Tumor tissues were immediately excised and minced by scissors and digested by $0.2 \%$ collagenase $/ 0.02 \%$ deoxyribonuclease solution to be single-cell suspensions. The XF96 Extracellular Flux Analyzer (Seahorse Bioscience, North Billerica, MA) was used to detect rapid, real-time changes in cellular glycolysis rate. Extracellular acidification rate (ECAR) 
was then measured according to the recommended protocol provided by the manufacturer [45]. Before measurements, 20,000 cells were cultured 6 hours in custom XF96 microplates and they were immersed in $200 \mu \mathrm{L}$ of unbuffered medium after being washed with the medium once, followed by incubation in the absence of $\mathrm{CO}_{2}$ for $1 \mathrm{~h}$. After measuring basal ECAR, oligomycin were introduced in real time. Because oligomycin-treated cells can only produce ATP via glycolysis, oligomycin provides a measure of maximum glycolytic potential. Analysis of ECAR reflects lactate excretion and serves as an indirect measure of glycolysis rate. Data are expressed as the mean \pm SEM of these values.

\section{X-ray irradiation}

The animals were restrained without anesthesia in a custom jig to be irradiated only on the tumor-bearing leg. Tumors were irradiated 5 Gy using an X-ray irradiator, XRAD-320 (Precision X-ray Inc., North Branford, CT). Imaging examinations were performed 24 hours after irradiation.

\section{Statistical analysis}

All results were expressed as the mean \pm SEM. The differences in means of groups were determined by 2-tailed Student's $t$ test. The minimum level of significance was set at $P<0.05$.

\section{Author contributions}

MM and HCK: devised the project, the main conceptual ideas and proof outline. MM and TK: worked out almost all of the technical details, and performed the numerical calculations and data analysis for the experiment with help from KS and JM. SK and KS: verified the numerical results by an independent implementation. ND: worked out image processing of EPR oximetry and provided technical support on both EPR and 13C-MRI experiment. MM, TK, and SK: wrote the manuscript with support from JBM and MCK. All authors discussed the results and commented on the manuscript.

\section{ACKNOWLEDGMENTS}

The authors thank Melissa Stauffer, $\mathrm{PhD}$, of Scientific Editing Solutions for providing editorial assistance.

\section{CONFLICTS OF INTEREST}

The authors have no potential conflicts of interest to disclose.

\section{FUNDING}

This work was supported by the Intramural Research Program, Center for Cancer Research, National Cancer Institute, National Institutes of Health. The funders had no role in study design, data collection and analysis, decision to publish, or preparation of the manuscript.

\section{REFERENCES}

1. Horsman MR, Nordsmark M, Overgaard J. Techniques to assess the oxygenation of human tumors. State of the art. Strahlenther Onkol. 1998; 174:2-5.

2. Stratford IJ, Adams GE, Bremner JC, Cole S, Edwards HS, Robertson N, Wood PJ. Manipulation and exploitation of the tumour environment for therapeutic benefit. Int J Radiat Biol. 1994; 65:85-94.

3. Maxwell PH, Dachs GU, Gleadle JM, Nicholls LG, Harris AL, Stratford IJ, Hankinson O, Pugh CW, Ratcliffe PJ. Hypoxia-inducible factor-1 modulates gene expression in solid tumors and influences both angiogenesis and tumor growth. Proc Natl Acad Sci U S A. 1997; 94:8104-9.

4. Hockel M, Schlenger K, Aral B, Mitze M, Schaffer U, Vaupel P. Association between tumor hypoxia and malignant progression in advanced cancer of the uterine cervix. Cancer Res. 1996; 56:4509-15.

5. Giaccia AJ. Hypoxic Stress Proteins: Survival of the Fittest. Semin Radiat Oncol. 1996; 6:46-58.

6. Palcic B, Skarsgard LD. Reduced oxygen enhancement ratio at low doses of ionizing radiation. Radiat Res. 1984; 100:328-39.

7. Dewhirst MW. Relationships between cycling hypoxia, HIF-1, angiogenesis and oxidative stress. Radiat Res. 2009; 172:653-65.

8. Matsumoto S, Hyodo F, Subramanian S, Devasahayam N, Munasinghe J, Hyodo E, Gadisetti C, Cook JA, Mitchell JB, Krishna MC. Low-field paramagnetic resonance imaging of tumor oxygenation and glycolytic activity in mice. J Clin Invest. 2008; 118:1965-73.

9. Yasui H, Matsumoto S, Devasahayam N, Munasinghe JP, Choudhuri R, Saito K, Subramanian S, Mitchell JB, Krishna MC. Low-field magnetic resonance imaging to visualize chronic and cycling hypoxia in tumor-bearing mice. Cancer Res. 2010; 70:6427-36.

10. Krishna MC, English S, Yamada K, Yoo J, Murugesan R, Devasahayam N, Cook JA, Golman K, ArdenkjaerLarsen JH, Subramanian S, Mitchell JB. Overhauser enhanced magnetic resonance imaging for tumor oximetry: coregistration of tumor anatomy and tissue oxygen concentration. Proc Natl Acad Sci U S A. 2002; 99:2216-21.

11. Matsumoto K, Subramanian S, Devasahayam N, Aravalluvan T, Murugesan R, Cook JA, Mitchell JB, Krishna MC. Electron paramagnetic resonance imaging of 
tumor hypoxia: enhanced spatial and temporal resolution for in vivo pO2 determination. Magn Reson Med. 2006; 55:1157-63.

12. Saito K, Matsumoto S, Yasui H, Devasahayam N, Subramanian S, Munasinghe JP, Patel V, Gutkind JS, Mitchell JB, Krishna MC. Longitudinal Imaging Studies of Tumor Microenvironment in Mice Treated with the mTOR Inhibitor Rapamycin. PLoS One. 2012; 7:e49456.

13. Ardenkjaer-Larsen JH, Fridlund B, Gram A, Hansson G, Hansson L, Lerche MH, Servin R, Thaning M, Golman K. Increase in signal-to-noise ratio of $>10,000$ times in liquidstate NMR. Proc Natl Acad Sci U S A. 2003; 100:10158-63.

14. Golman K, Ardenkjaer-Larsen JH, Petersson JS, Mansson S, Leunbach I. Molecular imaging with endogenous substances. Proc Natl Acad Sci U S A. 2003; 100:10435-9.

15. Day SE, Kettunen MI, Gallagher FA, Hu DE, Lerche M, Wolber J, Golman K, Ardenkjaer-Larsen JH, Brindle KM. Detecting tumor response to treatment using hyperpolarized 13C magnetic resonance imaging and spectroscopy. Nat Med. 2007; 13:1382-7.

16. Golman K, Zandt RI, Lerche M, Pehrson R, ArdenkjaerLarsen JH. Metabolic imaging by hyperpolarized 13C magnetic resonance imaging for in vivo tumor diagnosis. Cancer Res. 2006; 66:10855-60.

17. Albers MJ, Bok R, Chen AP, Cunningham CH, Zierhut ML, Zhang VY, Kohler SJ, Tropp J, Hurd RE, Yen YF, Nelson SJ, Vigneron DB, Kurhanewicz J. Hyperpolarized 13C lactate, pyruvate, and alanine: noninvasive biomarkers for prostate cancer detection and grading. Cancer Res. 2008; 68:8607-15.

18. Matsumoto S, Saito K, Yasui H, Morris HD, Munasinghe JP, Lizak M, Merkle H, Ardenkjaer-Larsen JH, Choudhuri R, Devasahayam N, Subramanian S, Koretsky AP, Mitchell JB, et al. EPR oxygen imaging and hyperpolarized 13C MRI of pyruvate metabolism as noninvasive biomarkers of tumor treatment response to a glycolysis inhibitor 3-bromopyruvate. Magn Reson Med. 2012.

19. Nelson SJ, Kurhanewicz J, Vigneron DB, Larson PE, Harzstark AL, Ferrone M, van Criekinge M, Chang JW, Bok R, Park I, Reed G, Carvajal L, Small EJ, et al. Metabolic imaging of patients with prostate cancer using hyperpolarized $\left[1-{ }^{13} \mathrm{C}\right]$ pyruvate. Sci Transl Med. 2013; 5:198ra108.

20. Aggarwal R, Vigneron DB, Kurhanewicz J. Hyperpolarized 1-[13C]-Pyruvate Magnetic Resonance Imaging Detects an Early Metabolic Response to Androgen Ablation Therapy in Prostate Cancer. Eur Urol. 2017; 72:1028-29.

21. Cunningham CH, Lau JY, Chen AP, Geraghty BJ, Perks WJ, Roifman I, Wright GA, Connelly KA. Hyperpolarized 13C Metabolic MRI of the Human Heart: Initial Experience. Circ Res. 2016; 119:1177-82.

22. Hendriksen EM, Span PN, Schuuring J, Peters JP, Sweep FC, van der Kogel AJ, Bussink J. Angiogenesis, hypoxia and VEGF expression during tumour growth in a human xenograft tumour model. Microvasc Res. 2009; 77:96-103.

23. Wilson CB, Lammertsma AA, McKenzie CG, Sikora K, Jones T. Measurements of blood flow and exchanging water space in breast tumors using positron emission tomography: a rapid and noninvasive dynamic method. Cancer Res. 1992; 52:1592-7.

24. Bentzen SM. Theragnostic imaging for radiation oncology: dose-painting by numbers. Lancet Oncol. 2005; 6:112-7.

25. Weissleder R. Molecular imaging: exploring the next frontier. Radiology. 1999; 212:609-14.

26. Herschman HR. Molecular imaging: looking at problems, seeing solutions. Science. 2003; 302:605-8.

27. Matsuo M, Miwa K, Tanaka O, Shinoda J, Nishibori H, Tsuge Y, Yano H, Iwama T, Hayashi S, Hoshi H, Yamada J, Kanematsu M, Aoyama H. Impact of [11C]methionine positron emission tomography for target definition of glioblastoma multiforme in radiation therapy planning. Int J Radiat Oncol Biol Phys. 2010; 82:83-9.

28. Matsuo M, Miwa K, Shinoda J, Kako N, Nishibori H, Sakurai K, Yano H, Iwama T, Kanematsu M. Target definition by C11-methionine-PET for the radiotherapy of brain metastases. Int J Radiat Oncol Biol Phys. 2009; $74: 714-22$.

29. Park HJ, Griffin RJ, Hui S, Levitt SH, Song CW. Radiationinduced vascular damage in tumors: implications of vascular damage in ablative hypofractionated radiotherapy (SBRT and SRS). Radiat Res. 2012; 177:311-27.

30. Brasch RC. New directions in the development of MR imaging contrast media. Radiology. 1992; 183:1-11.

31. Brasch RC, Shames DM, Cohen FM, Kuwatsuru R, Neuder M, Mann JS, Vexler V, Mühler A, Rosenau W. Quantification of capillary permeability to macromolecular magnetic resonance imaging contrast media in experimental mammary adenocarcinomas. Invest Radiol. 1994; 29:S8-11.

32. Mole DR, Blancher C, Copley RR, Pollard PJ, Gleadle JM, Ragoussis J, Ratcliffe PJ. Genome-wide association of hypoxia-inducible factor (HIF)-1alpha and HIF-2alpha DNA binding with expression profiling of hypoxiainducible transcripts. J Biol Chem. 2009; 284:16767-75.

33. Xia X, Lemieux ME, Li W, Carroll JS, Brown M, Liu XS, Kung AL. Integrative analysis of HIF binding and transactivation reveals its role in maintaining histone methylation homeostasis. Proc Natl Acad Sci U S A. 2009; 106:4260-5.

34. Semenza GL. Defining the role of hypoxia-inducible factor 1 in cancer biology and therapeutics. Oncogene. 2010; 29:625-34.

35. Papandreou I, Cairns RA, Fontana L, Lim AL, Denko NC. HIF-1 mediates adaptation to hypoxia by actively downregulating mitochondrial oxygen consumption. Cell Metab. 2006; 3:187-97.

36. Feron O. Pyruvate into lactate and back: from the Warburg effect to symbiotic energy fuel exchange in cancer cells. Radiother Oncol. 2009; 92:329-33.

37. Jaffray DA. Image-guided radiotherapy: from current concept to future perspectives. Nat Rev Clin Oncol. 2012; 9:688-99. 
38. Devasahayam N, Subramanian S, Murugesan R, Cook JA, Afeworki M, Tschudin RG, Mitchell JB, Krishna MC. Parallel coil resonators for time-domain radiofrequency electron paramagnetic resonance imaging of biological objects. J Magn Reson. 2000; 142:168-76.

39. Devasahayam N, Subramanian S, Murugesan R, Hyodo F, Matsumoto K, Mitchell JB, Krishna MC. Strategies for improved temporal and spectral resolution in in vivo oximetric imaging using time-domain EPR. Magn Reson Med. 2007; 57:776-83.

40. Subramanian S, Devasahayam N, Murugesan R, Yamada K, Cook J, Taube A, Mitchell JB, Lohman JA, Krishna MC. Single-point (constant-time) imaging in radiofrequency Fourier transform electron paramagnetic resonance. Magn Reson Med. 2002; 48:370-9.

41. Matsumoto K, English S, Yoo J, Yamada K, Devasahayam N, Cook JA, Mitchell JB, Subramanian S, Krishna MC. Pharmacokinetics of a triarylmethyl-type paramagnetic spin probe used in EPR oximetry. Magn Reson Med. 2004; 52:885-92.

42. Saito K, Matsumoto S, Devasahayam N, Subramanian S, Munasinghe JP, Morris HD, Lizak MJ, Ardenkjaer-Larsen JH, Mitchell JB, Krishna MC. Transient decrease in tumor oxygenation after intravenous administration of pyruvate. Magn Reson Med. 2011; 67:801-7.

43. Dharmaraj CD, Thadikonda K, Fletcher AR, Doan PN, Devasahayam N, Matsumoto S, Johnson CA, Cook JA, Mitchell JB, Subramanian S, Krishna MC. Reconstruction for time-domain in vivo EPR 3D multigradient oximetric imaging - a parallel processing perspective. Int J Biomed Imaging. 2009; 2009:528639.

44. Matsumoto S, Saito K, Takakusagi Y, Matsuo M, Munasinghe JP, Morris HD, Lizak MJ, Merkle H, Yasukawa K, Devasahayam N, Suburamanian S, Mitchell JB, Krishna MC. In vivo imaging of tumor physiological, metabolic, and redox changes in response to the anti-angiogenic agent sunitinib: longitudinal assessment to identify transient vascular renormalization. Antioxid Redox Signal. 2014; 21:1145-55.

45. Wu M, Neilson A, Swift AL, Moran R, Tamagnine J, Parslow D, Armistead S, Lemire K, Orrell J, Teich J, Chomicz S, Ferrick DA. Multiparameter metabolic analysis reveals a close link between attenuated mitochondrial bioenergetic function and enhanced glycolysis dependency in human tumor cells. Am J Physiol Cell Physiol. 2007; 292:C125-36. 